\title{
A neurosurgical challenge: awake mapping in "critical” language area tumours
}

\author{
Mihaela Cosman ${ }^{1}$, Ionut Mihail Pantiru², Bogdan Florin Iliescu ${ }^{1,2}$, \\ Nina Straticiuc ${ }^{3}$, lulia Aldea ${ }^{4}$, Vlad Buraga ${ }^{4}$, \\ Gabriela Florenta Dumitrecu ${ }^{5}$, Ion Poeata ${ }^{1,2}$
}

1 "Gr. T. Popa" University of Medicine and Pharmacy of Iași, Department of Neurosurgery, ROMANIA

2 "N. Oblu" Emergency Clinical Hospital, Iași, Department of Neurosurgery, ROMANIA

3 "N. Oblu" Emergency Clinical Hospital, Iași, Department of Anaesthesia and Intensive Care, RomanIA

4 "N. Oblu" Emergency Clinical Hospital, Iași, Department of Neurosurgery, Psychologist, ROMANIA

5 "N. Oblu" Emergency Clinical Hospital, Iași, Department of Anatomopathology, ROMANIA

\section{ABSTRACT}

Introduction. Despite the technological development lesion located in or near language area still represent a challenge for every neurosurgeon. Awake craniotomy and intraoperative neurophysiological monitoring come to our help. Different techniques variation exists among specialized centres. We present our experience and the set up for this procedure.

Materials and methods. We conducted a retrospective analysis of collected data from 10 patients with brain tumours located in or near language area to which we performed awake craniotomy and intraoperative neurophysiological monitoring. They were admitted in Third Department of Neurosurgery," Prof. Dr. N. Oblu" Emergency Clinical Hospital, Yassi, Romania, between January 2014 and July 2018.

Results. Presenting symptoms had a duration more than a month in $60 \%$ of patients. In $80 \%$ of them were represented by epileptic seizures and the rest of $20 \%$ had transient aphasia elements. The median age of presentation was 28 years old with a male dominance. The histological reports indicated: fibrillary astrocytoma $-40 \%$, anaplastic astrocytoma - 30\%, oligodendroglioma - $20 \%$ and metastases $-10 \%$. Gross total resection was performed in half of the cases and subtotal in just one case, in which the spontaneous speech and object naming showed repeated impairment in time of tumour debulking. The surgical intervention was well tolerated by all the patients. The intensity of cortical stimulation used was between $4-10 \mathrm{~mA}$. Postoperatively two patients had neurological aggravation, with full recovery at 3 months follow up period, two were stationary and six had symptoms remission. Conclusion. A young age of presentation, a paucity of symptoms, the chance for an increase in overall survival and progression free survival impose the need for direct

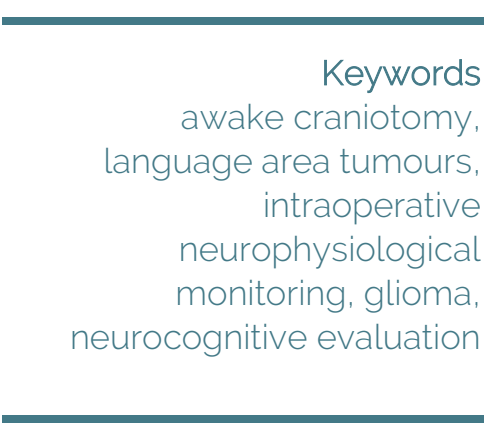

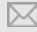

Corresponding author: Mihaela Coșman

Gr. T. Popa" University of Medicine and Pharmacy of lași, Department of Neurosurgery, Romania

mihaelacosman@yahoo.com

Copyright and usage. This is an Open Access article, distributed under the terms of the Creative commons Attribution Non-Commercial No Commons Attribution Non-Commercial No
Derivatives License (https://creativecommons org/licenses/by-nc-nd/4.0/) which permits noncommercial re-use, distribution, and reproduction in any medium, provided the original work is unaltered and is properly cited.

The written permission of the Romanian Society of Neurosurgery must be obtained for commercial re-use or in order to create a derivative work.

ISSN online 2344-4959 (C) Romanian Society of Neurosurgery

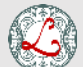

First published December 2019 by London Academic Publishing www.lapub.co.uk 
communication and feedback with the patient in time of tumour resection. Thus, awake craniotomy and intraoperative neurophysiological monitoring is the golden standard for selected cases of language area tumours.

\section{INTRODUCTION}

The role of surgery in the management of brain tumors is nowadays more and more prominent. This results from the fact that the extent of resection, e.g. in glioma cases is linked to increased survival rate and progression free survival [30]. The most challenging cases of them all are those located in and near eloquent areas, because of the high risk of producing new neurological deficit or worsen the previous ones, after surgery. For this reason, traditionally, the approach of those lesion was less invasive and with great caution [2, 11, 25].

To obtain the goal of a higher degree of resection with minimal neurological impairment, in language area surgery it is used the technique of awake craniotomy (AC) and intraoperative neurophysiological monitoring (IOM) $[7,17]$. This is helpful especially for those with preserved speech function like for example those manifested through epileptic seizures. For a proper selection of the cases, suitable for this technique, preoperative neurocognitive assessment is crucial and special battery of tests must be applied. $[5,15]$.

Despite the fact that the principals of those intraoperative techniques are the same worldwide there are some difference in implementing them. In this study we present our experience and method from surgical, anesthetic, neurocognitive and intraoperative brain mapping point of view.

\section{MATERIALS AND METHODS}

We conducted a retrospective analysis of collected data from 10 patients with brain tumors who underwent awake craniotomy and were admitted in the Third Department of Neurosurgery," Prof. Dr. N. Oblu" Emergency Clinical Hospital, Yassi, Romania, between January 2014 and July 2018. Inclusion criteria were: (1) patients diagnosed by contrast enhancement MRI with tumors located in or near language areas, (2) age > 18 years old, (3) preserved preoperative language function or slightly affected, (4) presented at 1 and respectively 6 months postoperative evaluation.

Every patient proposed for this surgical technique, from the neurosurgical point of view was evaluated by the psychologist and the anesthesiologist to confirm that it's completely eligible. Before the intervention they were prepared for 3 days, repeating and working with the psychologist on the items used intraoperatively. The initial assessment consisted in a battery of tests with an accent on reviling even the slit language dysfunction (spontaneous and automatic speech, word repetition, objects/images/forms naming and reading special texts from speech exercises). Other function tested include: executive function, praxis, memory, calculus and the writing.

The neuroanesthesia consisted in asleep-awakeasleep variant. After the induction step, the patient was positioned in a manner that allowed an easy access to the airway, comfortable for him and for the surgeon and also permitted communication with the psychologist. After the patient's head fixation, the skin incision was infiltrate with a mixt of lindocaine and epinephrine. When the craniotomy was finished and we started to open the dura mater the procedure of awakening the patient begun and throughout the tumor resection it was used total intravenous anesthesia (TIVA, Propofol -50 - $150 \mu \mathrm{g}$ $/ \mathrm{kgc} / \mathrm{min}$, the opioid used was Fentanyl 0, 5 $1 \mu \mathrm{g} / \mathrm{kgc} / \mathrm{h})$.

The neurocognitive intraoperative assessment was made by the same psychologist who evaluated the language function, testing patient's spontaneous speech, counting, object / images naming and depending on the case, limb muscle strength.

In all cases we performed direct cortical and subcortical brain stimulation with a bipolar probe (ball tips ends $5 \mathrm{~mm}$ apart, NimEclipse system, Medtronic) and we chose the high frequency technique. The start stimulation intensity was increased gradually with $0,5 \mathrm{~mA}$. We preferred to stimulate all the expose brain with the same intensity and after that to rise it. A site was considered positive if 2 of 3 stimulation lead to language tests dysfunction. The stimulation was repeated in time of resection, the subcortical pathways were tested more often at the end of debulking. The quantification of resection was made using intraoperative echography (Esaote system).

\section{RESULTS}

At the beginning of our study we identified a total of 58 cases of tumors located in or near language area, 
but only 10 of them have met all the criteria, the vast majority had important speech dysfunction (45 cases with motor or sensitive aphasia, in 2 cases we performed only biopsy and one patient was under 18 years old). The gander ratio showed male dominance; the median age distribution of the group was 28 years old. The clinical manifestation was represented by transient language disturbance with aphasia elements ( 2 patients) and epileptic seizures (8 patients). The symptoms duration was more than a month in $60 \%$ of cases. Other patient's characteristic are presented in Table 1.

\begin{tabular}{|l|l|l|l|l|l|l|l|l|}
\hline No. & Age & Gender & Location & Histology & $\begin{array}{l}\text { Degree of } \\
\text { resection }\end{array}$ & $\begin{array}{l}\text { Postop } \\
\text { evolution }\end{array}$ & $\begin{array}{l}\text { 6mo } \\
\text { Postop MRI } \\
+ \text { C }\end{array}$ & RCTX \\
\hline 1. & 66 & F & Left T & MTS & Total & Fav & no c.e. & + \\
\hline 2. & 46 & M & Left T & ODG III & NTR & Fav & no c.e. & + \\
\hline 3. & 37 & F & Left T & FA & STR & Stat & stat & - \\
\hline 4. & 27 & M & Left T & AA & GTR & Stat & no c.e. & + \\
\hline 5. & 37 & M & Left F & FA & NTR & Fav & stat,no rec & - \\
\hline 6. & 38 & M & Left T & ODG III & NTR & Fav & p.c.e. & + \\
\hline 7. & 20 & M & Left F & AA & GTR & Agg & p.c.e. & + \\
\hline 8. & 22 & F & Left T & AA & NTR & Fav & p.c.e. & + \\
\hline 9. & 28 & M & Right T & FA & GTR & Agg & no rec. & - \\
\hline 10. & 28 & M & Left F & FA & GTR & Fav & no rec & - \\
\hline
\end{tabular}

TABLE 1. Some characteristic of patients from the study: F- female, M-male, T-temporal lobe near/in Wernicke area, F-frontal lobe near/in Broca area, MTS- metastasis, ODG-oligodendroglioma, FA- fibrillary astrocytoma, AA- anaplastic astrocytoma, fav- favorable, stat- stationary, agg- aggravation, no c.e. - no contrast enhancement, p.c.e- peripheral contrast enhancement, no rec- no recurrence. RCTX-radio-chemotherapy.

a.

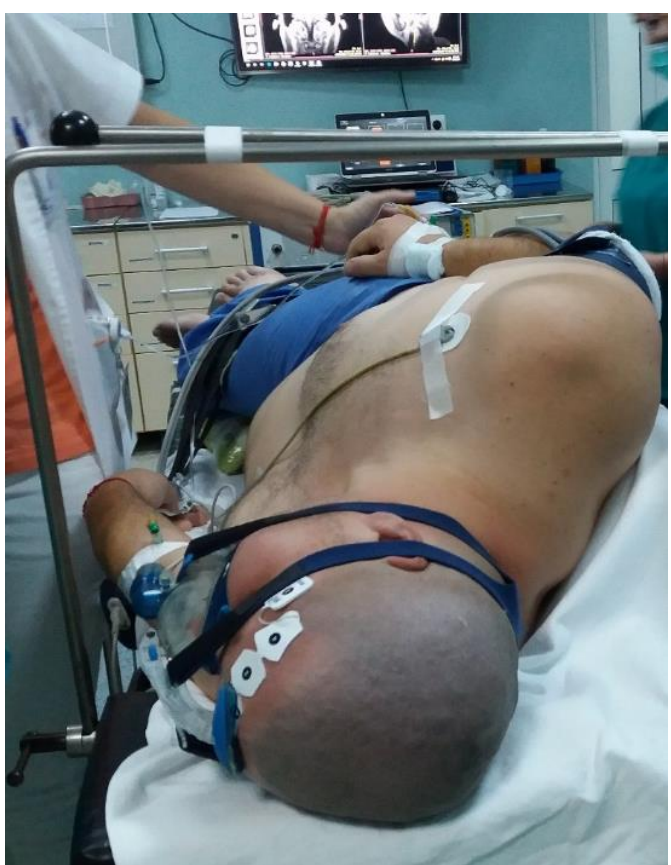

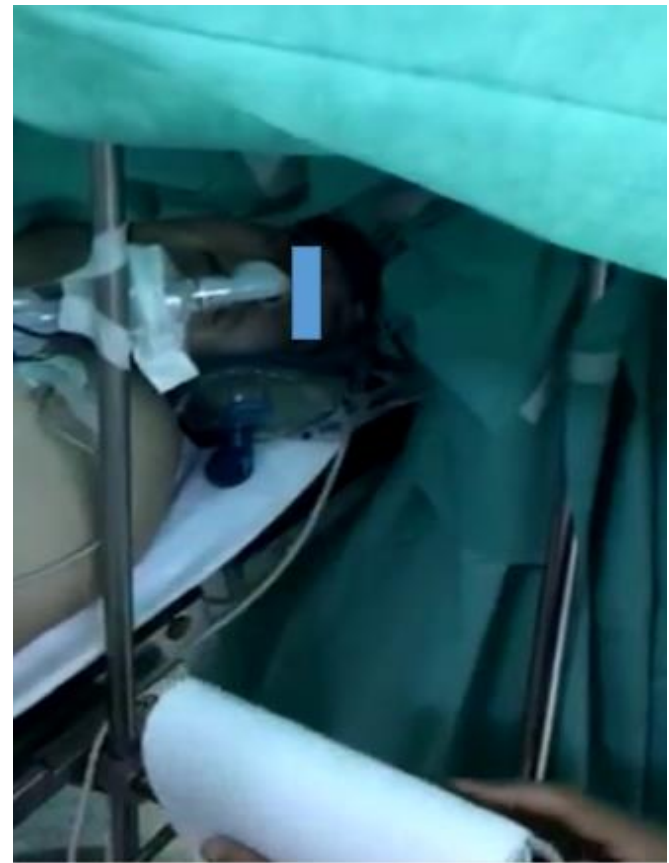

b. 
C.

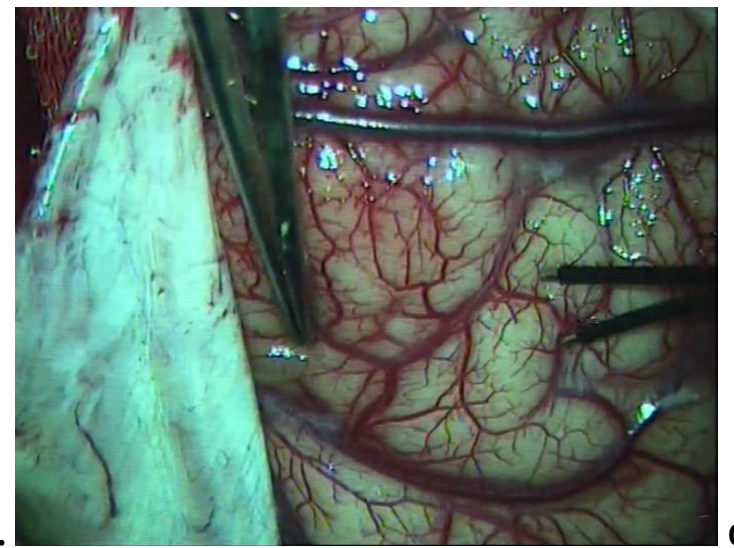

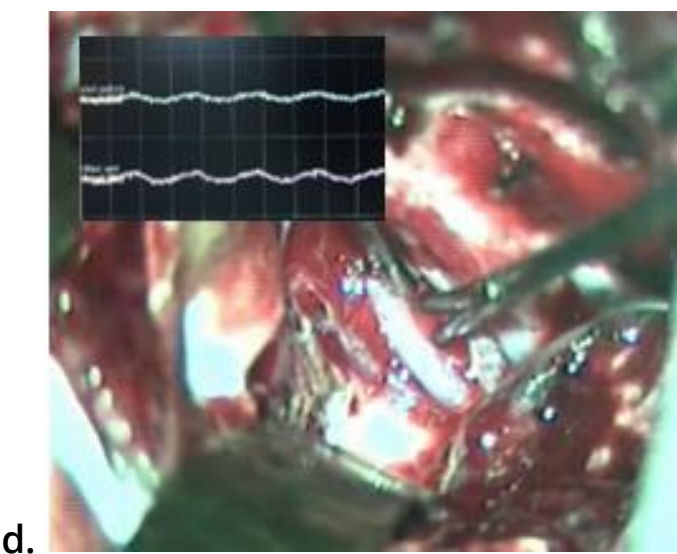

FIGURE 1. a. anesthesia and patient's positioning; b. awake stage, psychologist showing images/cards for naming test; c. brain mapping - direct cortical stimulation with bipolar probe, choosing the site for cerebrotomy; d. deep tumor resection and subcortical stimulation of white matter tracts, no motor respons (upper left) on electomyographic registration of abductor policis and tibialis anterior muscle (NimEclipse, Medtronic).

All patient tolerated well the surgery. The stimulation intensity used was between 4-10mA. In one case, in time of tumor resection we registered language dysfunction with tests performance decreasing. No intraoperative seizures were found. The interval from starting the process of awakening and proper communication was between 20 and 40 minutes. In Figure 1 are presented the intraoperative steps of the intervention, from anesthesia to cortical mapping and tumor debulking.

In two cases we registered postoperative neurologic aggravation as follow: one patient had transient sensitive aphasia elements which remitted completely until discharge, under anti-edematous drugs administration and another case manifested with motor aphasia which needed logopedic therapy. At 6 moths' evaluation the resolution of symptoms was completed.

All patients were monitored al list one year postoperatively (clinical and imagistic). Two of our cases first enrolled in the study presented neurologic deterioration associated with imagistic sign of recurrence and needed reoperation, at 4 and respectively 5 years after the first intervention. The histological reports reveal tumor progression from anaplastic astrocytoma to glioblastoma. Recurrence with no surgical solution was found in one case of grade III ODG, at three and a half years evaluation.

\section{DISCUSSION}

Maximizing the extent of resection and minimizing the postoperative neurological and neurocognitive disturbance becomes nowadays mandatory in the surgical treatment of brain tumors $[6,27]$. Due to oncological impact on overall survival and progression free survival in low-grade glioma (LGG) cases some suggested an early surgical approach or resection when those lesions are discovered incidentally [21]. To support this statement Duffau presented the idea of supratotal cytoreduction in diffuse gliomas. Even though the study included only 15 patient and the follow up period was short it was registered a reduction in the malignant transformation rate compared with the group of only complete resection [40]. Another proposal from Kamp et al. consisted in the supramarginal resection of metastases. The cases in which it was practiced this technique had a one-year local recurrence rate of $29,1 \%$ compared with $58,6 \%$ for those with only gross total resection [22].

Although there are some controversies about the modern treatment of high-grade-glioma (HGG) cytoreductive surgery still represents a key element in their management $[1,3,16]$. This is shown by Lacroix et al. how observed on a study of 416 glioblastoma (GB) that the reduction of tumor volume more than $98 \%$ is associated with a significant survival advantage [24]. Other study on 500 glioblastomas from 2011 published Sanai et al. noticed a significant increase in survival rate $(p<0$, 0001) even when the degree of resection was $78 \%$ [32].

It's well known that oligodendroglial tumors have an increase sensitivity to chemotherapy and there is 
a development of the oncological treatment with introduction of radiotherapy and immunotherapy in their protocols, however the first step in the management of those lesion is still represented by surgical resection. Studies shows that more than $90 \%$ of tumor volume reduction is associated with increase in the overall survival and progression free survival. Having a relatively good prognostic, the quality of life and the neurological status must be one of the neurosurgeon priorities [35, 38, 39].

To achieve such a goal, we need improvement and development of techniques designed to help use us in evaluating and detecting in real time the functional brain tissue and to set the relationship with the lesion. Another reason to implement those methods lays in the fact that brain tumors with a high incidence are usually located in or near eloquent areas. One example is represented by LGGs which are usually found in those locations, because of chemoarchitectonic and cytoarchitectonic similarities $[13,28]$. The third most common gliomas - oligodendroglioma are located with predilection in those regions as well (frontal lobe $50-65 \%$, temporal lobe $45 \%$, parietal lobe 7-20\%) [35]. Although our number of patients is small, there is a dominance from the histological point of view of LGGs (i.e., fibrillary astrocytoma, 40\%) followed by high-gradeglioma (HGG i.e. anaplastic astrocytoma, 30\%) and oligodendroglioma (20\%), matching the literature data.

There is a shift from imagine - guided surgery (intraoperative functional MRI and neuronavigation) towards functional - guided interventions (awake craniotomy and intraoperative neuropsychological monitoring). This is because neuroimaging concept is based on reduction only not taking into account the functional feedback. Preserving the classical anatomical landmarks for eloquent cortex, which have a wide variability interindividual is not enough. Also, it is hard to differentiate primary critical region from secondary or compensatory ones whose resection wont produces permanent neurological or neurocognitive deficits, thus reducing the degree of resection and the chance of prolonged free survival [12]. Hence the utility of combining the neurocognitive assessment during awake craniotomy and the result form direct cortical/subcortical mapping (DCM).

The history and the development of awake craniotomy starts in 1886 and was done by Victor
Horsley, first being used in epileptic surgery. Special anesthetic protocol are needed for this type of surgery and different variants are available by now. The improvement and introduction to asleep-awakeasleep (AAA) method was performed by Penfield, Ingvar and Hall in 1950, later Hansen described and used the awake-awake-awake anesthesia. The third option is represented by asleep-awake variant [36, 41]. Our anesthetic team chose and utilized the AAA variant.

Even though the number of patient with lesion located near/in language area was initially 58 only 10 meet all the inclusion criteria. Because of the complexity and potential risks of this procedure the contraindication must be evaluate wisely. Of the relative ones we enumerate: neurological causes: aphasia, confusion, agitation and cognitive disorders; psychiatric diseases; highly vascular tumors; airway problems; history of difficult intubation, obstructive sleep apnea; morbid obesity $[23,41]$. For these reasons in our practice, before deciding to perform this procedure, the psychologist and the anesthetist evaluate the case too and together discuss the eligibility for this type of intervention.

While awake craniotomy provides a close communication and an accurate evaluation, there is still room for other functional intraoperative tools. In a study published in 2010 de Benedictis et al. it is shown that the association between awake craniotomy and DCM increases the extent of resection and secondary the overall survival in patients with LGGs. This is achieved by resecting all the affected tissue up to critical individual limits with no need for safety margin around the eloquent area $[10,11]$. Kelm et al. evaluated 61 gliomas located in language area in which it was performed awake craniotomy and observed that GTR was higher in the patient operated and with IOM (61, 7\% vs. $28,6 \%)$. Beside the maintaining the integrity of the eloquent cortex, conservation of the subcortical pathways and tracts it's even more important because in this part of the brain the potential for neuroplasticity and reshaping is much smaller comparing to the cortical surface. Damaging the white matter tracts increases the risk for permanent neurological deficits. Although the preoperative imaging modalities have developed and we have many information about de language function localization and about the cortical and subcortical network involvement in this process, 
mapping remains an essential step along with awake craniotomy in the surgical management of language area tumors [7, 18, 20, 23].

The advantage of using both methods was reflected in the degree of resection of our cases too. So, because we had a real time assessment of neurologic and neurocognitive performances, we could achieve gross total resection in half of the patients, with more confidence in the postoperative functional and imagistic status. This had a favorable impact especially for fibrillary astrocytoma cases because: first of all the patients were young (28 yo) whose quality of life was an extremely important outcome factor and secondly the risk of malignant transformation was reduced. Those cases were followed up 2 years with no signs of recurrence.

Still in the light of quality of life preservation and because the admission symptoms were epileptic seizure in $90 \%$ of cases, the maintenance of language integrity was essential. Therefore, in one case were decided to perform only subtotal resection. It's about a 37 years old female, with the histological report of fibrillary astrocytoma, in which we had intraoperative language disturbance manifested by speech blockage and even sensitive aphasia when the tumor margins were stimulated and further resect was attempted.

Until now we talked about the role and advantages of an extensive resection, but this dogma should not be obtained by sacrificing the functional status. In 2015 Duffau published an article where is presented the multistage surgical resection. The idea is based on the action of neuroplasticity process. A functional reorganization and the chance for a new intervention without postoperative permanent deficits, in individual cases may be obtain. The reshaping may be induced by the surgery itself and other contributing factor may be represented by the rehabilitation therapy and tumor growth $[8,14]$.

It's important to know when is the proper time for the patient to stat the testing. Meskelevicius et al. published in 2019 a study in which it's shown the strong correlation between the speed of reaction and the patient's age and the fact that the speed of reaction it's at list two times slower than in the preoperative period, being the slowest in the first 1020 minute after the awaking. Although we had a small number of cases none of them failed and we could perform the awake surgery in all. Our patients started to react better after minimum of 20 minutes after awakening. During resection the psychologist communicated and evaluated continuously the language and motor function. We didn't find the positive cortical sites for language in all the cases. One possible reason must be the fact that the size of the craniotomy wasn't very big and so the exposed surface of the brain was limited. Another plausible reason is represented by the neural reshaping induced by the neuroplasticity process and so the imagistic landmarks for the eloquent area didn't match with the cortical ones. Some surgeon prefers a larger exposer to find the eloquent cortical sites not basing on these negative language sites $[26,29]$.

The stimulation technique and the value of stimulus parameters varies between different studies. The discrepancy comes from the fact that there are an inter- and intra-individual factor which determine this, for instance: tumor histological type and symptoms duration [9]. Usually a biphasic current it is used, the intensity it's raised gradually with 0,5 or $1 \mathrm{~mA}$ at a maximum of $10 \mathrm{~mA}$. To find the threshold which generates the response and not to generate the after-discharge byproducts it's a matter of perseverance, skill and a challenge [19, 31, 37]. We used the same parameters and in situation were no response was found we didn't raise the intensity higher than $10 \mathrm{~mA}$, because of the increased risk for seizure induction, especially in the patients with these symptoms

In meta-analysis published by Satoer et al in which he evaluated the studies about the possibility of cognitive preservation in eloquent area glioma surgery, in the postoperative period, language function was found altered in seven out of nine studies. At 3 months follow up period five out of six studies have revealed improvement in language deficit to a preoperative level $[4,34]$. The percentage of postoperative deficit reposts varies: Sanai found a rate of $14 \%$ - new speech deficit, and $8,4 \%$ aggravation; Duffau noticed a high degree of impairment 33\%-100\%, but with no severe permanent disability [11, 33]. In our cases postoperative speech deterioration were observed in two patients but full recovery was obtained after logopedic therapy. One of them remitted the symptoms even before discharge and the other one at 6 months evaluation returned to the initial stat. 


\section{CONCLUSIONS}

There is a diversity of histological types of tumors located in eloquent areas of the brain, some of them have a relatively slow growth pattern allowing the neuroplasticity process to act. For this reason, the patients presenting symptoms are just mild dysfunction instead of severe problems as in the cases of rapid growth rate tumors. With this in mind and with the chance for a prolonged overall survival, the importance of quality of life impose the necessity for a balanced decision between the degree of resection and the possibility of new postoperative neurologic deficits.

Despite the obvious development of preoperative functional evaluation only a direct approach and communication with the patient through tumor removal may increase the chances for a good postoperative functional status outcome and a higher degree of resection. Awake craniotomy and intraoperative neurophysiological monitoring meet those criteria and are the golden standard for selected cases of 'critical' language area tumors.

\section{Disclosures}

The authors report no conflict of interest concerning the materials or methods used in this study or the findings specified in this paper.

\section{REFERENCES}

1. Abrudan C, Cocis A, Cernea D, Suciu B, Cheptea M, Florian $\mathrm{SI}$. Surgery of high-grade gliomas - pros in favor of maximal cytoreductive surgery. Romanian Neurosurgery, 18(1): 38 53, 2011.

2. Antonsson $M$, Longoni $F$, Jakola $A$, Tisell $M$, Thordstein $M$, Hartelius L. Pre-operative language ability in patients with presumed low-grade glioma. J Neurooncol, 137(1):93-102, 2018.

3. Baritachii A, Gubian A, Florian SI. The impact of surgery in high grade gliomas - a literature review. Romanian Neurosurgery, XXIX 3: 291 - 304, 2015.

4. Barzilai O, Moshe BS, Sitt R, Sela G, Shofty B, Ram Z. Improvement in cognitive function after surgery for lowgrade glioma. J Neurosurg, 1:1-9, 2018.

5. Bondari S, Bondari D, Pîrşcoveanu M, Moroşanu DV, Muşetescu AE, Tudorică V, Pîrşcoveanu DFV. Study on cognitive decline in patients diagnosed with brain tumors. Rom J Morphol Embryol, 58(4):1185-1192, 2017.

6. Bogaciu NS, Teleanu D, Ciurea AV. New technologies for low-grade glioma surgery. Romanian Neurosurgery, 33(2), 122-126, 2019.

7. Chang WH, Pei YC, Wei KC, Chao YP, Chen MH, Yeh HA, Jaw FS, Chen PY: Intraoperative linguistic performance during awake brain surgery predicts postoperative linguistic deficits. Journal of Neuro-Oncology, 139:215-223, 2018.

8. Charras P, Herbet G, Deverdun J, de Champfleur NM, Duffau $H$, Bartolomeo P, Bonnetblanc F. Functional reorganization of the attentional networks in low-grade glioma patients: A longitudinal study. Cortex, 63: 27 - 41, 2015.

9. Corley JA, Nazari P, Rossi VJ, Kim NC, Fogg LF, Hoeppner TJ, Stoub TR, Byrne RW. Cortical stimulation parameters for functional mapping. Seizure. , 45:36-41, 2017.

10. De Benedictis A, Moritz-Gasser S, Duffau H. Awake mapping optimizes the extent of resection for low-grade gliomas in eloquent areas. Neurosurgery. 66(6):1074-84, 2010.

11. Duffau $\mathrm{H}$. Is non-awake surgery for supratentorial adult low-grade glioma treatment still feasible? Neurosurg Rev, 41(1):133-139, 2018.

12. Duffau H. A new philosophy in surgery for diffuse lowgrade glioma (DLGG): Oncological and functional outcomes. Neurochirurgie, 59: 2-8, 2013.

13. Duffau H, Capelle L. Preferential brain location of low-grade glioma Comparison with glioblastomas and review of hypothesis. Cancer, 100(12):2622-6, 2004.

14. Duffau $H$, Taillandier L. New concepts in the management of diffuse low-grade glioma: Proposal of a multistage and individualized therapeutic approach. Neuro-Oncol 17(3), 332-342, 2015.

15. Dwan TM, Ownsworth T, Chambers S, Walker DG, Shum DHK. Neuropsychological assessment of individuals with brain tumor: comparison of approaches used in the classification of impairment. Front.Oncol, 5:56, 2015.

16. Flechl B, Sax C, Ackerl M, Crevenna R, Woehrer A, Hainfellner J, Preusser M, Widhalm G, Kiesel B, LütgendorfCaucig C, Dieckmann K, Steffal C, Marosi C, Hassler MR. The course of quality of life and neurocognition in newly diagnosed patients with glioblastoma. Radiother Oncol, 125(2):228-233, 2017.

17. Gerritsen JKW, Lidia Arends L, Klimek M, Dirven CMF,Vincent AJPE. Impact of intraoperative stimulation mapping on high-grade glioma surgery outcome: a metaanalysis. Acta Neurochir, 161(1): 99-107, 2019.

18. Ghinda CD, Duffau H. Network Plasticity and Intraoperative Mapping for Personalized Multimodal Management of Diffuse Low-Grade Gliomas. Front Surg, 4:1-8, 2017.

19. Gollwitzer $S$, Hopfengärtner R, Rössler K, Müller T, Olmes DG, Lang J, Köhn J, Onugoren MD, Heyne J, Schwab S, Hamer HM. Afterdischarges elicited by cortical electric stimulation in humans: When do they occur and what do they mean? Epilepsy Behav., 87:173-179, 2018.

20. Ille S, Engel L, Kelm A, Meyer B, Krieg SM. LanguageEloquent White Matter Pathway Tractography and the Course of Language Function in Glioma Patients. Front Oncol., 8:572, 2018.

21. Jakola AS, Skjulsvik AJ, Myrmel KS, Sja ${ }^{\circ}$ vik $K$, Unsga ${ }^{\circ}$ rd $G$, Torp SH, Aaberg K, Berg T,Dai HY, Johnsen K, Kloster R, Solheim O. Surgical resection versus watchful waiting in low-grade gliomas. Annals of Oncology, 28: 1942-1948, 2017. 
22. Kamp MA, Dibué $M$, Niemann L, Reichelt DC, Felsberg J, Steiger HJ, Szelényi A, Rapp M, Sabel M. Proof of principle: supramarginal resection of cerebral metastases in eloquent brain areas. Acta Neurochir (Wien). , 154(11):1981-6, 2012.

23. Kelm A, Sollmann N, Ille S, Meyer B, Ringel F, Krieg SM. Resection of Gliomas with and without Neuropsychological Support during Awake Craniotomy-Effects on Surgery and Clinical Outcome. Front Oncol. , 7:176, 2017.

24. Lacroix M, Abi-Said D, Fourney DR, Gokaslan ZL, Shi W, DeMonte F, Lang FF, McCutcheon IE, Hassenbusch SJ, Holland E, Hess K, Michael C, Miller D, Sawaya R. A multivariate analysis of 416 patients with glioblastoma multiforme: prognosis, extent of resection, and survival. J Neurosurg., 95(2):190-8, 2001.

25. Leal RTM, Barcellos BM, Landeiro JA.Technical Aspects of Awake Craniotomy with Mapping for Brain Tumors in a Limited Resource Setting. World Neurosurg, 113:67-72, 2018.

26. Meskelevicius D, Schäfer A, Weber JK, Hegmann L, Haddad L, Kamp MA, Mainzer B, Rapp M, Steiger HJ, Sabel M. Determination of optimal time window for cortical mapping in awake craniotomy: assessment of intraoperative reaction speed. Neurosurg Rev. 15, 2019

27. Monticelli M, Zeppa P, Zenga F, Altieri R, Mammi M, Bertero L, Castellano I, Cassoni P, Melcarne A, La Rocca G, Sabatino G, Ducati A, Garbossa DThe post-surgical era of GBM: How molecular biology has impacted on our clinical management. A review. Clin Neurol Neurosurg., 170:120126, 2018.

28. Oslobanu A, Florian SI. Anatomic location in high grade gliomas. Romanian Neurosurgery, XXIX 3: 269 - 275, 2015

29. Pallud J, Rigaux-Viode O, Corns R, Muto J, Lopez Lopez C, Mellerio C, Sauvageon X, Dezamis E. Direct electrical bipolar electrostimulation for functional cortical and subcortical cerebral mapping in awake craniotomy. Practical considerations. Neurochirurgie. 63(3):164-174, 2017.

30. Roelz R, Strohmaier D, Jabbarli R, Kraeutle R, Egger K,
Coenen AV, Weyerbrock A, Reinacher PC. Residual tumor volume as best outcome predictor in low grade glioma - a nine-year near-randomized survey of surgery vs. biopsy. Scientific Reports, 6:32286, 2016.

31. Saito T, Muragaki Y, Maruyama T, Tamura M, Nitta M, Okada Y. Intraoperative Functional Mapping and Monitoring during Glioma Surgery. Neurol Med Chir (Tokyo)., 55 Suppl 1:1-13, 2015.

32. Sanai N, Berger MS. Extent of resection influences outcomes for patients with gliomas. Rev Neurol (Paris). 167(10):648-54, 2011.

33. Sanai N, Mirzadeh Z, Berger MS. Functional outcome after language mapping for glioma resection. N Engl J Med, 358:18-27, 2008.

34. Satoer D, Visch-Brink E, Dirven C, Vincent A. Glioma surgery in eloquent areas: can we preserve cognition? Acta Neurochir (Wien). 158(1):35-50, 2016.

35. Simonetti G, Gaviani P, Botturi A, Innocenti A, Lamperti E, Silvani A. Clinical management of grade III oligodendroglioma. Cancer Manag Res., 27(7):213-23, 2015.

36. Sitnikov AR, Grigoryan YA, Mishnyakova LP. Awake craniotomy without sedation in treatment of patients with lesional epilepsy. Surg Neurol Int., 9:177, 2018.

37. So EL, Alwaki A. A guid for cortical electrical stimulation mapping. J Clin Neurophysiol, 35: 98-105, 2018.

38. Thon N, Kreth FW, Tonn JC. The role of surgery in grade II/III oligodendroglial tumors. CNS Oncol, 4(5), 317-323, 2015.

39. Torensma R. The Dilemma of Cure and Damage in Oligodendroglioma: Ways to Tip the Balance Away from the Damage. Cancers (Basel), 10(11): 431, 2018.

40. Yordanova YN, Duffau H. Supratotal resection of diffuse gliomas - an overview of itsmultifaceted implications. Neurochirurgie, 63(3):243-249, 2017.

41. Zhang K, Gelb AW. Awake craniotomy: indications, benefits, and techniques. Rev Colomb Anestesiol., 46(Supp):46-51, 2018. 